\title{
Hüttner
}

Grundzüge der Marktforschung 
Prof. Dr. Manfred Hüttner

\section{Grundzüge der Marktforschung}

Ein Leitfaden für Studium und Praxis

mit 107 Beispielen

Zweite, durchgesehene und ergänzte Auflage

Betriebswirtschaftlicher Verlag Dr. Th. Gabler - Wiesbaden 


\section{Vorwort zur 1. Auflage}

Das vorliegende Buch ist unmittelbar entstanden aus Vorlesungen und Ubungen für Studierende der oberen Semester einer Höheren Wirtschaftsfachschule. Wenn es somit auch in erster Linie den Bedürfnissen der Ausbildung von Führungskräften der Wirtschaft (im Sinne des „middle management"), also stark praxisbetonten Zwecken, dient und dienen soll, so hat es doch auch darüber hinausgehende Ziele. Bei einer Durchsicht der vorliegenden Literatur, die keineswegs dürftig, sondern im Gegenteil recht umfangreich ist, zeigt sich nämlich, daß zwar eine Fülle von Schriften zu Einzelfragen vorliegt, dagegen noch kaum eine in deutscher Sprache verfaßte zusammenfassende, lehrbuchmäßige Darstellung, die eine Orientierung über die Gesamtheit der inzwischen schon recht weit differenzierten einzelnen Sachgebiete bzw. Methoden der Marktforschung gibt. Nicht zuletzt dürfte dieser Mangel auch darauf zurückzuführen sein, daß der Bereich der Marktforschung noch immer in der Bundesrepublik kaum Gegenstand der Lehre und Forschung an den Universitäten ist und wohl aus diesem Grunde vielfach eine wissenschaftliche Systematik bei der Behandlung von Fragen der Marktforschung nicht in Erscheinung tritt.

Obwohl also dem Buch nach Ursprung und Anlage an sich wissenschaftliche Ambitionen fremd sind, wird doch hier und da angesichts der Situation auf dem Gebiet der Marktforschung und des Zieles einer umfassenden und systematischen Darstellung gelegentlich die Erörterung von Grundsatzfragen bzw, von einzelnen noch ungelösten Problemen vonnöten sein. In diesem Sinne mag das Buch auch als ein Beitrag zur Entwicklung einer wissenschaftlichen Systematik auf dem Sektor der Marktforschung und damit zur Herausbildung dieses Gebietes als eigene wissenschaftliche Disziplin gewertet werden und somit auch für an der Marktforschung wissenschaftlich interessierte Kreise, insbesondere aber die zur Marktforschung neigenden Studenten der Betriebs- bzw. Volkswirtschaftslehre, von Bedeutung sein. Andererseits wird es infolge seiner stark praxisbetonten Zielsetzung und der Behandlung unter rein betriebswirtschaftlichen Aspekten über den engeren Kreis der Studierenden hinaus sicher auch unter den Praktikern der Wirtschaft Leser finden.

Dieser stark praxisbetonten Zielsetzung entsprechend hat der Verfasser bewußt auf die Heranziehung fremdsprachiger und damit oft schwer zugänglicher Literatur weitgehend verzichtet und sich bemüht, in großem Umfange Beispiele zu den einzelnen Problemen zu bringen, diese Beispiele aber nicht zu konstruieren, sondern sie in der Praxis durchgeführten Lösungen zu entnehmen. Daß dies in sehr vielen Fällen möglich war, verdankt der Verfasser zahlreichen Marktforschungsinstituten und Verlagen, die die Arbeit durch Uberlassung von Material bzw. die Erlaubnis zum Abdruck unter- 
stützten; allen, die das Zustandekommen des Buches auf diese oder vielfältige sonstige Weise förderten, sei an dieser Stelle nochmals herzlich gedankt.

Schließlich sei darauf hingewiesen, daß es bei dem Versuch einer umfassenden und dabei doch kurzen und prägnanten Darstellung der einzelnen Probleme wohl nicht ausbleiben kann, daß die eine oder andere Frage nur gestreift wird. Dabei mag es geschehen sein, daß im Einzelfall dem jeweiligen Problem vielleicht nicht das richtige Gewicht beigemessen worden ist; für sachliche Kritik in dieser und anderer Richtung ist der Verfasser jederzeit dankbar.

Siegen, im April 1965 


\section{Vorwort zur 2. Auflage}

Erfreulicherweise hat das Buch eine außerordentlich weite Verbreitung gefunden, nicht nur in der Praxis und dem ursprünglichen Adressatenkreis, den damaligen Höheren Wirtschaftsfachschulen und heutigen Fachhochschulen, sondern vor allem auch an den Universitäten. Das mußte bei der Vorbereitung einer Neuauflage zu der Frage führen, ob nicht - neben der Aktualisierung - die Grundkonzeption geändert werden müsse, und zwar in mehrfacher Richtung: Einmal hätte das mathematisch-statistische Instrumentarium viel ausführlicher dargestellt werden können (z. B. hinsichtlich der Stichprobentheorie oder der Faktorenanalyse bzw. der multivariaten Methoden generell), zum anderen wäre eine Erweiterung im Hinblick auf grundlegende Fragestellungen, z. B. die Rolle und Bedeutung der Marktforschung im gegenwärtigen Wirtschaftssystem überhaupt, die Marktforschung für „öffentliche Güter" bzw. grundsätzlich im ,non-profit-Sektor", Markt- und Bedarfsforschung in sozialistischen Ländern, speziell der DDR, in Erwägung zu ziehen gewesen. Weiter hätte eine stärkere Einbeziehung der ausländischen, insbesondere auch anglo-amerikanischen Literatur erfolgen können und schließlich eine Zusammenstellung von "Fragen und Aufgaben" nach jedem Abschnitt.

Wenn eine solche grundlegende Neubearbeitung und Erweiterung unterblieben ist, so einerseits deshalb, weil die weite Verbreitung des Buches gerade zu beweisen scheint, daß die bisherige (,lehrbuchartige") Konzeption die richtige gewesen ist, andererseits aus ganz pragmatischen Gründen: Eine grundlegende Utberarbeitung wäre sowohl beim Verfasser als auch beim Verlag auf zeitliche Schwierigkeiten gestoßen, was - insbesondere auch wegen des dann notwendig werdenden Neusatzes des gesamten Textes eine ganz wesentliche Zeitverzögerung bedeutet hätte.

Die Gesamtdarstellung ist deshalb im wesentlichen unverändert gelassen worden. Im laufenden Text wurden lediglich noch verbliebene Fehler — nach der Ausmerzung der meisten im Nachdruck 1969 - berichtigt. Einige (gröBere) Aktualisierungen sind in einem Nachtrag (S. $341 \mathrm{ff}$.) zusammengestellt; im Text erfolgt, mit besonders gekennzeichneten (*) Anmerkungen, Verweis hierauf. Bei geringerem Umfang findet sich die inhaltliche Darstellung sofort in der Anmerkung (und nur eine Zusammenstellung - damit der Gesamtheit der Ergänzungen - am Schluß). Als Nachtrag ist auch einige inzwischen erschienene neuere Literatur, unter Einschluß von Veröffentlichungen aus der DDR einerseits, den USA usw. andererseits, angegeben. Ebenso 
werden hier Neuauflagen von im Text zitierten - und entsprechend dem dargestellten Prinzip dort unverändert gelassenen - Werken genannt. Den Abschluß bildet eine Art „Aufgabensammlung", eine Zusammenstellung von Fragen und Aufgaben, die im Prinzip der Gliederung des Stoffes folgt.

Manfred Hüttner 


\section{Inhaltsverzeichnis}

Einführung

Die Entwicklung der Marktforschung

Erster Teil

\section{Das Wesen der Marktforschung}

A. Gegenstand der Marktforschung.......... 21

B. Arten der Marktforschung. . . . . . . . . . . . . 22

1. Demoskopische und ökoskopische Marktforschung . . . . . . . . 22

2. Instituts- und betriebliche Marktforschung . . . . . . . . . . . 23

3. Betriebswirtschaftliche und volkswirtschaftliche Marktforschung . 24

4. Primäre und sekundäre Marktforschung . . . . . . . . . . . 25

5. Binnen- und Auslandsmarktforschung . . . . . . . . . . . . . 26

6. Konsumgüter- und Investitionsgütermarktforschung (sowie Marktforschung für Dienstleistungen) . . . . . . . . . . 27

7. Prospektive und retrospektive Marktforschung . . . . . . . . . 28

8. Beschaffungs- und Absatzmarktforschung . . . . . . . . . . . 28

9. Introduktions- und Okonomisierungsmarktforschung . . . . . . 29

C. Abgrenzung der Marktforschung. . . . . . . . . . 29

1. Marktforschung und Markterkundung . . . . . . . . . . . . . 29

2. Marktforschung, Marktanalyse und Marktbeobachtung . . . . . . 30

3. Marktforschung und Absatzforschung . . . . . . . . . . . . . 30

4. Marktforschung und Verbrauchsforschung . . . . . . . . . . 31

5. Marktforschung, Meinungsforschung und Motivforschung . . . . 32

6. Zusammenfassung ................. 34

$$
\text { Zweiter Teil }
$$

Die Methoden der Marktforschung

Abschnitt I

Die Methoden der demoskopischen Marktforschung

A. Die Befragung . . . . . . . . . . . . . . . 35

I. Die (normale) Umfrage . . . . . . . . . . . . . 36 
1. Methodische Grundprobleme der Umfrage . . . . . . . . . 36

a) Die Arten der Befragung . . . . . . . . . . . . . . . . 36

(1) Unterscheidung nach dem befragten Personenkreis . . 36

(2) Unterscheidung nach der Art der Erhebung . . . . . . 38

(3) Unterscheidung nach der Zahl der Untersuchungsthemen 42

b) Die Arten des Interviews . . . . . . . . . . . . . . 43

c) Die Arten der Fragestellung . . . . . . . . . . . . . . 45

(1) Die direkte Befragung . . . . . . . . . . . . . . . 45

(2) Die indirekte Befragung . . . . . . . . . . . 45

(a) Psychotaktische Befragungsweise . . . . . . . . 46

(b) Psychologische Tests . . . . . . . . . . . . . . . 47

Exkurs: Sonstige Techniken der Motivforschung . . . . . 53

d) Die Arten der Fragen . . . . . . . . . . . . . . 54

(1) Einteilung nach der Antwortmöglichkeit . . . . . . . 54

(a) Offene Fragen . . . . . . . . . . . . . . . . 55

(b) Geschlossene Fragen . . . . . . . . . . . . 56

(aa) Alternativfragen . . . . . . . . . . . . . . 57

(bb) Mehrfach-Auswahl-Fragen (Selektivfragen) . . 60

(2) Einteilung nach der Verwendung von Vorlagen . . . . 66

(3) Einteilung nach dem Zweck . . . . . . . . . . . . . 67

(a) Instrumentelle Fragen . . . . . . . . . . . . . . 68

(b) Ergebnisfragen . . . . . . . . . . . . . . . . . . . . 69

e) Die Arten der Auswahl der Befragten . . . . . . . . . 69

(1) Die Zufallsauswahl (Randomverfahren) . . . . . . . 71

(a) Die Normalform der uneingeschränkten

Zufallsauswahl ........... . . 72

(b) Die Sonderformen . . . . . . . . . . . . . . . . 74

(2) Die Quotenauswahl . . . . . . . . . . . 77 
2. Die technische Durchführung der Umfrage . . . . . . . . 83

a) Die Gewinnung des Materials . . . . . . . . . . . 83

(1) Die Vorbereitung der Befragung . . . . . . . . . . 83

(a) Definition und Klärung des Problems . . . . . . . 83

(b) Durchführung einer Leitstudie . . . . . . . . . . 84

(c) Aufstellung des Forschungsplanes . . . . . . . . . 84

(d) Entwicklung des Fragebogens . . . . . . . . . . 85

(aa) Die Utbersetzung der „Programmfrage“ in "Testfragen“............. . 85

(bb) Die Formulierung der Fragen . . . . . . . . 86

(cc) Die Reihenfolge der Fragen . . . . . . . . . . 87

(dd) Der Umfang des Fragebogens . . . . . . . . 87

(ee) Die „Probebefragung“ . . . . . . . . . . . . 87

(e) Festlegung der Befragungsmasse . . . . . . . . . . . 88

(aa) Fehlerspanne und Sicherheitsgrad . . . . . . 88

(bb) Der Umfang der Stichprobe . . . . . . . . . 92

(2) Die Durchführung der Befragung . . . . . . . . . 94

(a) Die Interviewer-Organisation . . . . . . . . . . 95

(b) Der Einsatz der Interviewer-Organisation . . . . 96

(aa) Vorbereitung des Interviewer-Einsatzes . . . . 96

(bb) Durchführung des Interviewer-Einsatzes . . . 97

(cc) Kontrolle des Interviewer-Einsatzes . . . . . 99

b) Die Aufbereitung des Materials . . . . . . . . . . . . . 100

(1) Die Aufbereitungsverfahren . . . . . . . . . . . 100

(a) Manuelle Verfahren . . . . . . . . . . . . . 100

(b) Maschinelle Verfahren . . . . . . . . . . . . . . 101

(2) Die Verschlüsselung . . . . . . . . . . . . . 103

c) Darstellung und Analyse des Materials . . . . . . . . 105

(1) Die Auswertung . . . . . . . . . . . . . 105

(a) Darstellung durch absolute oder relative Zahlen . . 106

(b) Vornahme von Signifikanzprüfungen . . . . . 108 
(c) Darstellung in ein- oder mehrgliedrigen Tabellen . 109

(d) Durchführung von Korrelations- und

Regressionsanalysen.......... 111

(2) Die Abfassung des Forschungsberichts . . . . . . . . 114

(a) Bestandteile des Forschungsberichts . . . . . . . . 114

(b) Inhalt des Forschungsberichts . . . . . . . . . 115

II. Das Panel . . . . . . . . . . . . . . . . . . . . . . . . . . 115

1. Begriff des Panels . . . . . . . . . . . . . . . . . . 115

2. Arten des Panels . . . . . . . . . . . . . . . . . . . . 116

a) Händler-Panels . . . . . . . . . . . . . . . . . 116

b) Verbraucher-Panels . . . . . . . . . . . . . . . . . . 117

c) Spezial-Panels . . . . . . . . . . . . . . . . . . . 118

3. Methodische Probleme des Panels . . . . . . . . . . . . . 119

a) Die Zusammensetzung des Panels . . . . . . . . . . . . 119

b) Der „Panel-Effekt" . . . . . . . . . . . . . . . 120

4. Technische Durchführung des Panels . . . . . . . . . . . . 121

a) Allgemeines zur technischen Durchführung . . . . . . . . 121

b) Aufbau und Durchführung wichtiger Arten des Panels . . 122

(1) Das allgemeine Einzelhandels-Panel . . . . . . . . . 122

(2) Das Haushalts-Panel für Verbrauchsgüter . . . . . . 123

(3) Das Haushalts-Panel für Gebrauchsgüter . . . . . . . 124

5. Bedeutung des Panels . . . . . . . . . . . . . . . . . . 124

Exkurs: Die Tendenzfrage . . . . . . . . . . . . . . . 125

B. Die Beobachtung. . . . . . . . . . . . . . 126

I. Begriff der Beobachtung . . . . . . . . . . . . . . 126

II. Arten der Beobachtung . . . . . . . . . . . . . . . . . . . . 127

1. Teilnehmende und nicht-teilnehmende Beobachtung . . . . . 127

2. Feld- und Laboratoriums-Beobachtung . . . . . . . . . . . 127

III. Methodische Probleme der Beobachtung . . . . . . . . . . . . 128

1. Die Zusammensetzung der Beobachtungsgesamtheit . . . . . 128

2. Der „Beobachtungs-Effekt" . . . . . . . . . . . . . . . . 129 
IV. Technische Durchführung der Beobachtung . . . . . . . . . . . 129

V. Bedeutung der Beobachtung . . . . . . . . . . . . 130

C. Das Experiment................... 131

I. Begriff des Experiments . . . . . . . . . . . . . . 131

II. Arten des Experiments . . . . . . . . . . . . . . . . . . . 132

1. Beobachtungs- und Befragungs-Experiment . . . . . . . . 132

2. Feld- und Laboratoriums-Experiment . . . . . . . . . 132

III. Methodische Probleme des Experiments . . . . . . . . . . . . 133

1. Das Problem der Versuchsanlage . . . . . . . . . . . . 133

2. Das Problem der kleinen Stichproben . . . . . . . . . . . 134

IV. Technische Durchführung des Experiments . . . . . . . . . . 135

V. Bedeutung des Experiments . . . . . . . . . . . . . 136

\section{Abschnitt II \\ Die Methoden der ökoskopischen Marktforschung}

A. Die Verfahrender Sammlung des Materials... . 137

I. Methodische Grundprobleme . . . . . . . . . . . . 137

1. Sammlung von Material und "Sammelmethode“ . . . . . . . 137

2. Die Quellen des Materials . . . . . . . . . . . . . . . 139

a) Primäre Quellen . . . . . . . . . . . . . . . . . 139

b) Sekundäre Quellen . . . . . . . . . . . . . . 140

(1) Externe Informationen . . . . . . . . . . . . 140

(2) Interne Informationen . . . . . . . . . . . . . . . 148

II. Technische Durchführung . . . . . . . . . . . . . . . . . . 149

1. Grundsätze der technischen Durchführung . . . . . . . . 149

2. Beispiele für die technische Durchführung von

Untersuchungen aufgrund der „Sammelmethode“ . . . . . . 151 
B. Die Verfahren der Auswertung des Materials. . 154

I. Okoskopische Methoden unter Anwendung einfacher statistischer Verfahren . . . . . . . . . . . . 155

1. Marktanteilsberechnungen . . . . . . . . . . . . . 155

a) Begriff des Marktanteils und Probleme seiner Ermittlung . 155

b) Regionale und zeitliche Analyse des Marktanteils . . . . : 157

(1) Die regionale Analyse . . . . . . . . . . . . . . 157

(2) Die zeitliche Analyse . . . . . . . . . . . . . . 158

c) Marktanteil und Feldanteil . . . . . . . . . . . . . . 159

2. Die Heranziehung von Marktindikatoren . . . . . . . . . . 159

a) Die Ermittlung von Marktindikatoren . . . . . . . . . . 159

(1) Allgemeine und spezielle Marktindikatoren . . . . . . 159

(2) Kaufkraftkennziffern . . . . . . . . . . . . . . . . 160

b) Die Anwendung von Marktindikatoren . . . . . . . . . 164

3. Elastizitätsberechnungen . . . . . . . . . . . . . . 166

a) Begriff der Elastizität und Probleme ihrer Ermittlung . . 166

b) Die Berechnung wichtiger Arten der Elastizität . . . . . . 167

(1) Preiselastizität . . . . . . . . . . . . . . . 167

(2) Verbrauchselastizität . . . . . . . . . . . . . 168

(3) Substitutionselastizität . . . . . . . . . . . . . . 169

4. Abnehmergruppenanalyse und Retroanalyse . . . . . . . 171

II. Die Heranziehung herkömmlicher statistisch-mathematischer

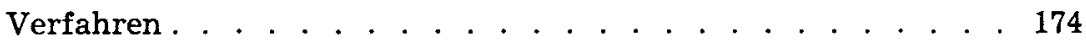

1. Häufigkeitsanalyse . . . . . . . . . . . . . . . . . 174

a) Die einfache Analyse der Häufigkeitsverteilung . . . . . . 174

b) Häufigkeitsanalyse im Wahrscheinlichkeitsnetz . . . . . . 176

2. Zeitreihenanalyse . . . . . . . . . . . . . . . . 178

a) Die Berechnung des Trends . . . . . . . . . . . . . . . 179

(1) Das Wesen der Trendprojektion . . . . . . . . . . 179

(2) Die Anwendung einzelner Arten des Trends . . . . . 180

(a) Linearer Trend . . . . . . . . . . . . . . . . . 180

(b) Logarithmischer Trend . . . . . . . . . . . . . 183

(c) Parabolischer und hyperbolischer Trend . . . . . . 184

(d) Logistischer Trend . . . . . . . . . . . . . . . 184

(3) Die Problematik der Trendberechnung . . . . . . . . 188 
b) Die Feststellung von Absatzrhythmen . . . . . . . . . . 188

(1) Das Problem der Konjunkturschwankungen . . . . . 189

(2) Saisonschwankungen . . . . . . . . . . . . . . . 191

(a) Schwankungen innerhalb des Jahres . . . . . . . 191

(b) Monats-, Wochen- und Tagesschwankungen . . . . 194

3. Regressions- und Korrelationsanalyse . . . . . . . . . . . 195

III. Die Heranziehung moderner statistisch-mathematischer

Verfahren . . . . . . . . . . . . . . . . 200

1. Die Anwendung ökonometrischer Modelle in der Marktforschung . . . . . . . . . . . . . . . 201

2. Die Anwendung der Input-output-Analyse in der Marktforschung . . . . . . . . . . . . . . . . 202

3. Die Anwendung von Operations-Research-Methoden in der Marktforschung . . . . . . . . . . . . . . . . . . 204

4. Die Anwendung spieltheoretischer Lösungsansätze in der Marktforschung ............... 206

\section{Dritter Teil}

Sachgebiete der betriebswirtschaftlichen Marktforschung

A. Absatzmarktforschung . . . . . . . . . . 207

I. Die Anwendungsgebiete der Absatzmarktforschung im allgemeinen . . . . . . . . . . . . . . . . 207

1. Untersuchung des Marktpotentials . . . . . . . . . . 207

a) Die Ermittlung der Größe, der Merkmale und der regionalen Differenzierung des Marktes . . . . . . . . . 207

(1) Größe und Merkmale des Marktes . . . . . . . . . 207

(2) Die regionale Differenzierung des Marktes . . . . . 211

b) Die Aufstellung von Verkaufsprognosen . . . . . . . . . 211

(1) Problematik und Bedeutung von Verkaufsprognosen . 211

(2) Prognosemethoden und ihre praktische Anwendung . . 213

(3) Die Entstehung der Verkaufsprognose . . . . . . . . 218

2. Untersuchung des absatzpolitischen Instrumentariums . . . . 219

a) Die Analyse der Preispolitik . . . . . . . . . . . . . 220

(1) Allgemeines . . . . . . . . . . . . . . . . 220

(2) Der Preistest . . . . . . . . . . . . . . . 221 
(a) Ermittlung der Vorstellungen über die Preishöhe . . 221

(b) Ermittlung der Aufnahmebereitschaft bei gegebenem Preis . . . . . . . . . . . . . . . 224

b) Die Analyse der Produktgestaltung . . . . . . . . . . . 226

(1) Allgemeines . . . . . . . . . . . . . . . 226

(2) Der Produkttest . . . . . . . . . . . . . . . . 226

(a) Der Test einzelner Seiten des Produkts . . . . . . 226

(b) Der Test des Produkts im ganzen . . . . . . . . . . 230

(c) Die technische Durchführung des Produkttests . . . 232

(3) Die Entwicklung neuer Erzeugnisse . . . . . . . . . 233

c) Die Analyse der Absatzmethoden . . . . . . . . . . . . 234

d) Die Analyse der Werbung . . . . . . . . . . . . . . . 235

(1) Werbeträgerforschung . . . . . . . . . . . . . 236

(a) Leserschaftsforschung . . . . . . . . . . . . . . 236

(b) Hörer- und Fernsehforschung . . . . . . . . . . 236

(2) Werbemittelforschung . . . . . . . . . . . . . 237

(a) Werbeerfolgsprognose . . . . . . . . . . . . 237

(a) Subjektive Methoden . . . . . . . . . . . . 238

(bb) Objektive Methoden . . . . . . . . . . . . 239

(b) Werbeerfolgskontrolle . . . . . . . . . . . . . 241

(a) Die Feststellung der Aufmerksamkeits- und

Gedächtniswirkung . . . . . . . . . 241

(bb) Die Feststellung der Handlungswirkung . . . 243

Exkurs: Noreensches Modell und IWEMA-Anzeigentest 245

(3) „Werbestatistik“ . . . . . . . . . . . . . . . 246

3. Die Einrichtung von Testmärkten . . . . . . . . . . . . . 246

a) Begriff des Testmarkt-Verfahrens . . . . . . . . . . . 246

b) Methodische Probleme . . . . . . . . . . . . . . . . . 247

c) Technische Durchführung . . . . . . . . . . . 249

d) Bedeutung . . . . . . . . . . . . . . . . . . . . . 249

Exkurs: Umfragen und gewerblicher Rechtsschutz . . . . . . . 250

II. Besondere Bereiche der Absatzmarktforschung . . . . . . . . 252

1. Die Marktforschung für Investitionsgüter . . . . . . . . . 253

a) Die Anwendung demoskopischer Methoden . . . . . . 253

(1) Befragung . . . . . . . . . . . . . . . . . . . 253

(2) Beobachtung und Experiment . . . . . . . . . . 257 
b) Die Anwendung ökoskopischer Methoden . . . . . . . . 258

(1) Verfahren der Sammlung des Materials . . . . . . 258

(2) Verfahren der Auswertung des Materials . . . . . . 258

2. Die Auslandsmarktforschung . . . . . . . . . . . . 261

a) Die Anwendung demoskopischer Methoden . . . . . . 262

(1) Befragung . . . . . . . . . . . . . . . . . . . . . 263

(2) Beobachtung und Experiment . . . . . . . . . . 264

b) Die Anwendung ökoskopischer Methoden . . . . . . . . 265

(1) Verfahren der Sammlung des Materials . . . . . . . 265

(2) Verfahren der Auswertung des Materials . . . . . . . 268

3. Die Marktforschung im Dienstleistungssektor . . . . . . . 272

a) Allgemeine Problematik der Marktforschung im Dienstleistungssektor . . . . . . . . . . . . . . 272

b) Marktforschung in einzelnen Bereichen des Dienstleistungssektors . . . . . . . . . . . . 273

(1) Marktforschung im Einzelhandel . . . . . . . . . . . 273

(2) Marktforschung im Versicherungs- und Bankgewerbe . 278

(3) Marktforschung im Fremdenverkehr . . . . . . . . . 280

(4) Marktforschung in sonstigen Bereichen des Dienstleistungssektors . . . . . . . . . . . . . 281

4. Die landwirtschaftliche Marktforschung . . . . . . . . . . 282

B. Sonstige Bereiche der Marktforschung . . . . . 282

1. Die Marktforschung auf dem Beschaffungsmarkt . . . . . . . . 282

a) Beschaffungsmarkt und Beschaffungsmarktforschung . . . . 282

b) Aufgaben der Beschaffungsmarktforschung . . . . . . . . . 283

c) Methoden und Quellen der Beschaffungsmarktforschung . . . 283

d) Besonderheiten der Marktforschung für international gehandelte Rohstoffe . . . . . . . . . . . . . 284

2. Die Marktforschung auf dem Arbeitsmarkt . . . . . . . . . . 285

a) Aufgaben der Arbeitsmarktforschung . . . . . . . . . . 286

b) Methoden der Arbeitsmarktforschung . . . . . . . . . . . . 287

(1) Die Anwendung demoskopischer Methoden . . . . . . . . 287

(2) Die Anwendung ökoskopischer Methoden . . . . . . . . . 287 
3. Die Marktforschung auf dem Finanzmarkt . . . . . . . . . . . 288

a) Aufgaben der Finanzmarktforschung . . . . . . . . . . . . 288

b) Methoden der Finanzmarktforschung . . . . . . . . . . . . 289

$$
\text { Vierter Teil }
$$

\section{Die Organisation der betriebswirtschaftlichen Marktforschung}

A. Die Träger der Marktforschung . . . . . . . . 291

I. Die betriebliche Marktforschung . . . . . . . . . . . . . . . 291

1. Wesen und Umfang der betrieblichen Marktforschung . . . . 291

2. Die Aufgaben der Marktforschungsabteilung im Betrieb . . . 295

3. Die organisatorische Stellung der Marktforschungsabteilung im Betrieb . . . . . . . . . . . . . . . . . . . . . . . 298

II. Die Instituts-Marktforschung . . . . . . . . . . . . . . . . . 302

1. Wesen und Arten der Instituts-Marktforschung . . . . . . . 302

2. Die Instituts-Marktforschung in der Bundesrepublik und im Ausland . . . . . . . . . . . . . . . . . . . 304

III. Sonstige Träger der Marktforschung . . . . . . . . . . . . . 307

1. Die Verbandsmarktforschung . . . . . . . . . . . . 307

2. Die Verbund-Marktforschung . . . . . . . . . . . . . 309

3. Die behördliche Marktforschung . . . . . . . . . . . . . 309

B. Die Kosten der Marktforschung . . . . . . . 310

I. Die Kosten für die Instituts-Marktforschung . . . . . . . . . 310

II. Die Kosten für die Marktforschung insgesamt . . . . . . . . . 313

C. Der Berufsstand des Marktforschers... . . . . . 315

I. Die Ausbildung des Marktforschers . . . . . . . . . . . . . . 315

II. Berufsverbände auf dem Gebiet der Marktforschung . . . . . . 318

Anhang

A. Merkblatt des DIVO-Instituts, Frankfurt a. M., zum Auswahlverfahren . . . . . . . . . . . . . . . . . . . 321

B. Quotenanweisung (Auswahlvorschrift) der EMNID-Institute, Bielefeld 323

C. Fragebogen der EMNID-Institute, Bielefeld . . . . . . . . . . . . 325

Nachtrag . . . . . . . . . . . . . . . . 341

Literaturverzeichnis . . . . . . . . . . . . . . . 353

Stichwortverzeichnis . . . . . . . . . . . . . . . 363 